\title{
sur la gestion et les finances publiques En Avril 2021
}

\section{BUDGET DE L'ÉtAT ET DES OPÉRATEURS}

\section{Données générales sur les finances publiques}

PIB : léger rebond au premier trimestre $2021(+0,4 \%)$

Dans une note du 30 avril 2021, I'INSEE indique qu'au premier trimestre 2021, le PIB augmente légèrement : $+0,4 \%$ après $-1,4 \%$ au quatrième trimestre 2020. Le rebond est cependant limité : le PIB demeure inférieur de $4,4 \%$ à son niveau d'avant crise sanitaire (fin 2019).

La production totale (biens et services) rebondit timidement : $+0,4 \%$, après $-0,6 \%$ au quatrième trimestre 2020. Néanmoins, la production de biens est en recul $(-1,0 \%$ après $+2,5 \%)$, notamment dans I'industrie manufacturière $(-1,2 \%$ après $+2,6 \%)$. En revanche, le secteur de la construction rebondit nettement $(+4,2 \%$ après - 2,2\%). La production de services marchands rebondit de manière plus modérée $(+0,3 \%$ après $-1,8 \%)$, ainsi que la production de services non marchands $(+1,0 \%$ après $-0,5 \%)$.

Les dépenses de consommation des ménages ne rebondissent que très légèrement ce trimestre (+0,3\%), après le net repli de 5,7\% au trimestre précédent, marqué par le deuxième confinement. L'investissement accélère ce trimestre $(+2,2 \%)$ mais la contribution des échanges extérieurs à la croissance du PIB est quant à elle négative $(-0,4 \%)$.

\section{Budget des pouvoirs publics}

Rapport de la Cour des comptes sur le budget de l'État 2020

Le 13 avril 2021, la Cour des comptes a rendu public son rapport sur le budget de l'État pour
I'année 2020, accompagné de 65 analyses de l'exécution budgétaire.

L'année 2020 a été marquée des interventions massives de l'État, qui se sont traduites par I'adoption de quatre lois de finances rectificatives. En exécution, le déficit s'est considérablement accru (+ $85 \mathrm{Md}$ ) par rapport à 2019, pour atteindre $178 \mathrm{Md}$ en 2020. En conséquence, la dette de l'État a connu une très forte progression (+ $178 \mathrm{Md}$ ) et atteint désormais $2001 \mathrm{Md}$.

Au-delà du budget général, la Cour a examiné I'ensemble des moyens financiers que l'État consacre aux politiques publiques et constate un suivi et un pilotage insuffisants. Elle formule cinq recommandations, dont quatre reprennent les recommandations déjà présentées dans son rapport sur le budget pour l'année 2019. Par sa recommandation nouvelle - et non des moindres - la Cour invite au plein respect du principe d'annualité budgétaire, et à n'ouvrir en lois de finances initiale et rectificative que les crédits nécessaires à l'exercice en cours et, sauf exception dûment justifiée, se conformer strictement à la règle de plafonnement des reports de crédits à $3 \%$ des crédits ouverts en application de l'article 15 de la LOLF.

La publication de ce rapport est réalisée conjointement au dépôt du projet de loi de règlement conformément à l'article 58 de la LOLF.

Certification des comptes de l'État pour I'année 2020

Le 13 avril 2021, la Cour des comptes a publié I'acte de certification des comptes de l'État.

La Cour certifie qu'au regard des règles et principes comptables applicables, le compte général de l'État de l'exercice 2020, arrêté le 2 avril 2021, est régulier et sincère, et donne une image fidèle de la situation financière et du 
patrimoine de l'État, sous quatre réserves substantielles:

1) La Cour a rencontré, à l'occasion de l'audit des comptes de 2020, des difficultés importantes pour conduire certaines de ses vérifications dans des conditions satisfaisantes. Elle n'est, dans plusieurs cas, pas en mesure de se prononcer sur le bien-fondé et la fiabilité des enregistrements comptables en raison tant des conditions de tenue de la comptabilité générale dans Chorus, que de l'organisation et du suivi du contrôle interne dans les ministères.

2) Plusieurs difficultés importantes, déjà relevées à l'issue des exercices précédents, continuent d'affecter la fiabilité des comptes. Elles se rapportent aussi bien au recensement et à l'évaluation des stocks et des matériels militaires de l'État qu'à l'évaluation d'une part significative de ses infrastructures civiles et militaires.

3) La Cour n'est pas en mesure de se prononcer avec une assurance raisonnable sur l'évaluation d'une part significative du patrimoine financier de l'État, en raison d'incertitudes ou de désaccords sur la valeur de cet actif.

4) Des insuffisances significatives affectent toujours le contrôle et l'enregistrement en comptabilité des données relatives aux charges de personnel, aux charges d'intervention et aux produits régaliens. Pour ces derniers, l'évaluation des créances sur les redevables ne donne pas une image fidèle des droits et obligations de l'État.

L'acte de certification est publié conjointement au dépôt du projet de loi de règlement conformément à l'article 58 de la LOLF.

\section{$\lambda$ Procédure budgétaire}

Présentation en Conseil des ministres du projet de loi de règlement 2020

Le 14 avril 2021, Le ministre de l'économie, des finances et de la relance, et le ministre délégué chargé des comptes publics, ont présenté, en Conseil des ministres, le projet de loi de règlement du budget et d'approbation des comptes de l'État (PLR) pour 2020. Cette présentation est marquée par un processus d'anticipation du calendrier budgétaire entamé en 2018 par le Gouvernement afin de faire bénéficier au Parlement d'un temps de débat plus long dédié aux résultats de l'exécution budgétaire et de la gestion. Présenté en Conseil des ministres en même temps que le programme de stabilité, il contribue au débat d'orientation des finances publiques. Ce "chaînage vertueux", auquel ont concouru le Parlement, la Cour des comptes et les administrations, facilitera l'exercice par le Parlement de sa mission d'évaluation des politiques publiques, dont l'approche a été renouvelée et s'inscrit en cohérence avec la démarche de "Printemps de l'évaluation » menée par l'Assemblée nationale depuis 2018.

Le projet de loi de règlement 2020 présente en particulier l'impact budgétaire de la crise sanitaire et économique et des mesures d'urgence prises tant sur les dépenses que sur les recettes de l'État. Il est complété d'éléments d'analyse issus des trois comptabilités de l'État, permettant de disposer d'une vision budgétaire et en comptabilité nationale, mais également d'une vision patrimoniale des comptes publics.

Au total, ce sont plus de $500 \mathrm{Md}$ qui ont été mobilisés par le Gouvernement, dont l'essentiel (478 Md€) sur le budget de l'État, pour faire face à la crise sanitaire. Le sous-secteur des administrations de sécurité sociale (ASSO) a porté près de $26 \mathrm{Md}$ de mesures, dont 11,8 Md€ de dépenses de santé exceptionnelles et 11,4 Md€ pour la prise en charge par I'Unedic de l'activité partielle. Les collectivités locales ont participé au financement du fonds de solidarité pour les entreprises à hauteur de 0,5 Md€.

Avis du Haut Conseil des finances publiques relatif au solde structurel des administrations publiques présenté dans le projet de loi de règlement de 2020

Dans son avis du 12 avril 2021, rendu public le 14 avril, le Haut Conseil des finances publiques (HCFP) constate que la crise sanitaire a conduit à des évolutions hors norme des finances publiques. Cette situation l'avait déjà amené à estimer, dans son avis sur le premier projet de loi de finances rectificatif pour 2020, que les circonstances exceptionnelles mentionnées à l'article 3 du Traité sur la stabilité, la coordination et la gouvernance (TSCG) étaient réunies.

Si ces évolutions ont rendu obsolète la loi de programmation en vigueur, le HCP rappelle que cette loi demeure la référence sur laquelle il doit s'appuyer pour rendre son avis.

Le HCFP note toutefois que la décomposition du solde public entre ses composantes exceptionnelles et temporaires, structurelle et conjoncturelle, est moins évidente qu'à l'accoutumée.

À cet égard, le HCFP relève que les modalités de calcul des mesures exceptionnelles et temporaires retenues par le Gouvernement conduisent à afficher une amélioration du solde structurel en 2020, en contradiction avec la très 
forte dégradation qui s'annonce persistante des finances publiques.

Dans cet avis, le HCFP prend toutefois acte que les déficits structurels qui lui sont ainsi soumis, estimés à 2,3 et 0,9 points de PIB respectivement pour 2019 et 2020, ne s'écartent pas de manière importante de la loi de programmation des finances publiques (LPFP) de janvier 2018, qui les prévoyait à 1,9 et 1,6 point de PIB respectivement. Le HCFP considère ainsi qu'il n'y a donc pas lieu de déclencher le mécanisme de correction prévu par l'article 23 de la loi organique du 17 décembre 2012 relative à la programmation et à la gouvernance des finances publiques.

\section{Budget des opérateurs}

Centre national de la recherche scientifique et sciences humaines et sociales

Dans un référé du 18 février 2021, publié le 13 avril 2021, la Cour des comptes souligne I'apport fondamental du CNRS aux unités de recherche dont il assure la co-tutelle. La Cour considère que la plus-value du CNRS est manifeste du fait de son implication dans le développement de son appui à l'internationalisation ou aux différents réseaux de recherche, ainsi qu'en termes de pilotage scientifique, grâce à la détermination partenariale d'objectifs scientifiques exigeants. Or, la Cour estime également que les infrastructures de soutien à la recherche universitaire en sciences humaines et sociales (SHS) ont besoin d'être renforcées. Les infrastructures de recherche en SHS sont en effet peu financées comparativement à leurs homologues d'autres disciplines scientifiques. Si la Cour relève que des efforts ont été consentis par le CNRS ou par les universités, elle constate qu'il est nécessaire désormais que le ministère leur apporte un soutien plein et entier.

Enfin, la Cour recommande de clarifier les modalités de recrutement des chercheurs du CNRS dans les disciplines de SHS afin de mettre un terme aux risques contentieux, deux concours de recrutement ayant été récemment annulés par les juridictions administratives au motif que les candidats avaient été écartés par le jury d'admissibilité sur des critères autres que ceux relatifs à la valeur scientifique et au mérite.

\section{Comptabilité PUbliQue}

\section{$\pi$ Règlementation comptable}

CNOCP : projet de norme sur les fonds propres pour le futur recueil de normes comptables destiné aux organismes de sécurité sociale

Le 23 avril 2021, le Conseil de normalisation des comptes publics (CNOCP) a publié son projet de norme $n^{\circ} 20$ sur les fonds propres. Cette norme définit les principales rubriques constituant les fonds propres des organismes de sécurité sociale et précise leurs principes comptables. Elle présente également les opérations de couverture des déficits cumulés des organismes de sécurité sociale par la Caisse d'amortissement de la dette sociale (CADES), prévues dans le cadre des Lois de financement de la sécurité sociale (LFSS).

\section{FinANCES LOCALES}

Bulletin d'Information Statistique, BIS n¹51: " La fiscalité directe locale en $2020 »$

Ce nouveau document de la DGCL livre de précieux enseignements sur cette terrible année 2020. Contrairement aux premiers chiffres parus annonçant mi-2020 des pertes fiscales colossales, la réalité est beaucoup plus nuancée. Le produit de la fiscalité directe des collectivités locales s'élève à 97,8 Md€ en 2020, en hausse de $+2,2 \%$ (après $+3,3 \%$ en 2019). Cette hausse s'explique principalement par les impôts économiques, qui ont progressé de $+3,0 \%$. Malgré tout, on peut considérer le verre à moitié vide et admettre que la hausse est beaucoup moins importante que l'année précédente $(+4,9 \%$ en 2019). Cette augmentation est tirée par la CFE qui augmente de $+3,2 \%$. A ce stade, deux éléments doivent être mentionnés. Les augmentations régulières de la CFE (indépendamment de la politique de taux) expliquent, en partie, le choix de la réforme des impôts de production votée par le législateur dans le cadre de la loi de finances pour 2021. Par ailleurs, en termes de CVAE, compte tenu du décalage de deux ans des bases fiscales, l'impact de la crise aura sans doute des effets plus marqués en 2021 et 2022.

Le produit des taxes "ménages » a moins augmenté $(+1,9 \%$ en 2020 , après $+2,9 \%$ ), hausse qui s'explique principalement par la revalorisation nominale votée tous les ans par le législateur.

Un dernier point d'information de cette note concerne le taux de taxe foncière qui a été pointé du doigt uniquement à charge de ces derniers mois. Sur 2020, la progression des taux du secteur communal a été très faible en 2020 
(+0,03 point). Au final, la progression des taux du foncier bâti sur l'ensemble du cycle électoral de 2014 a été, elle aussi, plus faible que sur les cycles précédents.

\section{Publication de la Dotation globale de fonctionnement (DGF)}

Le 2 avril 2021, la DGCL a rendu publics les montants de DGF avec les différentes parts sur le site dédié à cela. Pour la première fois, les données divulguées donnent lieu à une cartographie en ligne permettant de visualiser territoire par territoire le montant par habitant, la variation de la DGF entre 2020 et 2021, la part de la DGF dans les recettes réelles de fonctionnement et enfin la part de la péréquation dans la DGF.

Avec la stabilisation globale du montant de la DGF de l'ordre de $27 \mathrm{Mds} €$ et l'absence d'évolution majeure des périmètres de l'intercommunalité, les montants individuels alloués à chaque collectivité se stabilisent également davantage. Rappelons par ailleurs que l'évolution des montants de la DGF est principalement la conséquence des effets des évolutions de la population d'un territoire à un autre mais également du prélèvement des parts forfaitaires pour alimenter les parts péréquation.

\section{Bilan de la répartition de la DSIL en 2020} et prévisions 2021

Selon les informations communiquées par la DGCL, "au 31 décembre 2020, plus de 574 M€ de subventions avaient été accordées au bénéfice de 3357 projets d'investissement partout sur le territoire ». II apparaît ainsi une sous-consommation de cette dotation, ce qui peut paraître surprenant dans le contexte actuel. Il peut s'agir soit d'un effet du télétravail ou la conséquence d'un problème structurel liée à cette subvention.

Sur 2021, cette enveloppe exceptionnelle, qui fait partie intégrante du plan France Relance, se déploie sur les exercices 2020 et 2021 et s'ajoute aux enveloppes de $570 \mathrm{M} €$ ouvertes dans la loi de finances pour 2020 et dans la loi de finances pour 2021, à la dotation d'équipement des territoires ruraux ( $1 \mathrm{Md})$ ), ainsi qu'à l'enveloppe de $650 \mathrm{M} €$ dédiée à la rénovation énergétique des bâtiments communaux et intercommunaux, elle aussi mobilisée dans le cadre du plan France Relance.

Au-delà des chiffres communiqués, ces informations démontrent progressivement l'imprégnation d'une culture de la datavisualisation au sein du Gouvernement. Aux chiffres communiqués s'ajoute une cartographie par région démontrant les projets financés par collectivités.

\section{Situation financière des petites villes 2020-2021, Avril 2021}

L'Association des petites villes de France vient de rendre son rapport sur la situation financière des collectivités qu'elle regroupe. Pour ce rapport, les recettes de fonctionnement ont globalement diminué de $60 \%$ pour les petites villes, tandis que les dépenses de fonctionnement ont augmenté de $26 \%$, démontrant bien l'effet ciseaux de la crise actuelle. Si ce choc a été partiellement absorbé, cela s'explique, en partie, par le recul des dépenses d'investissement (- $56 \%$ ), même si la capacité d'autofinancement a fortement baissé, de l'ordre de $35 \%$. Les petites villes ont finalement peu recouru au dispositif d'étalement de charges et seulement $8 \%$ des petites villes ont activé l'acompte sur la clause de garantie des recettes. Dans le sondage mené par cette association d'élus locaux, les surcoûts liés à la crise sanitaire se poursuivent en 2021 pour les deux tiers des petites villes. En revanche, pour l'instant, les incertitudes demeurent sur le un rebond de l'investissement en 2021.

ADCF et banque des territoires, Baromètre de la commande publique 2021 résultats 2020

Cette traditionnelle analyse permet, en premier lieu, d'apprécier l'évolution des dépenses d'investissement sur le moyen terme. En effet, la commande publique de travaux a baissé de - 25\% entre 2020 et 2019 (soit un recul de 7 Mds€), tandis que les marchés de services représentent désormais la plupart des dépenses d'investissement, à hauteur de 48 Mds€. Les évolutions en 2020 sont très nuancées selon les types de dépenses. La commande publique diminue de façon globale de $18 \%$ avec des écarts selon les secteurs. Ainsi, en matière de bâtiments publics, la baisse est de l'ordre de $-18,5 \%$, contre $-12 \%$ dans le domaine de la santé sociale. Les aménagements connaissent la plus forte baisse (environ - 25,5\%, les dépenses évoluent de 4,3 à 3,2 Mds€). Il s'agit des opérations liées aux aménagements économiques (zones industrielles, zones artisanales, zones commerciales) et des aménagements urbains (Parkings, lotissements, infrastructures urbaines, aménagements de l'espace public). 


\section{FINANCES SOCIALES}

\section{$\lambda$ Situation des comptes sociaux et conséquences de la crise sanitaire}

Rapport de la Cour des Comptes sur le budget de l'État en 2020 : rapport « Plan d'urgence face à la crise sanitaire "

Dans son rapport sur l'exécution budgétaire 2020 , la Cour des Comptes a consacré un rapport particulier à la nouvelle mission "Plan d'urgence pour faire face à la crise ». Celle-ci a fait l'objet, à travers 4 lois de finances rectificatives, d'ouvertures de crédits d'un montant total de 70,5 Mds€, consommés en CP à hauteur de 41,82 Mds€, qui se répartissent en 4 programmes; le programme consacré à l'activité partielle, très rapidement mis en place, a été doté au total de 22,6 Mds€ et consommé à hauteur de 17, $8 \mathrm{Mds} €$ (report de 2,5 M€ sur 2021). Le programme dédié au Fonds de solidarité a été doté de 19,7 Mds€ et consommé pour 11,8 Mds€ (report intégral sur 2021), avec un rythme d'utilisation qui a été très impacté par les décisions du gouvernement sur son périmètre ; le programme " prises de participations exceptionnelles " a été doté de 20 Mds€ et utilisé à hauteur de 8,3 Mds€, au profit d'Air France, de la SNCF, d'EDF et du Fonds Ace Aero (la Cour s'interroge sur la pertinence de l'imputation à ce programme d'opérations concernant la SNCF et EDF, qui ne paraissent pas directement liées à la crise); enfin le programme " compensation à la Sécurité sociale des allègements de prélèvement sociaux » a été doté de 8,2 Mds€, dont seuls 3,9 Mds€ ont été dépensés, les crédits non consommés devant être reportés sur le programme "Fonds de solidarité ». La Cour, constatant que près de $40 \%$ des crédits ouverts vont être reportés sur 2021, s'interroge sur le réalisme des prévisions budgétaires initiales; elle note par ailleurs que la mission a été prolongée en 2021, mais que la nouvelle mission "Plan de relance " pourrait comporter des dépenses de soutien aux entreprises, et que la coexistence de supports budgétaires risque de nuire à la lisibilité des dépenses.

Rapport du comité de suivi d'aide aux entreprises confrontées à la crise du Covid 19

Le Comité de suivi et d'évaluation des mesures de soutien financier aux entreprises touchées par la crise sanitaire, institué par la première LFR de 2020, a remis son premier rapport d'étape le 19 avril. L'analyse porte sur $17 \mathrm{me-}$ sures de soutien, concentrées autour de 4 ensembles principaux : activité partielle, Fonds de solidarité, prêts garantis et report d'échéances fiscales et sociales. Après avoir constaté qu'au regard des résultats économiques en 2020, la France se situait dans la moyenne européenne (sauf pour le commerce extérieur, très dégradé), le comité relève que le recours aux aides a été largement fonction de la taille des entreprises, les petites entreprises représentant, dans les montants versés, une part supérieure à la proportion des effectifs qu'elles emploient, les aides qu'elles ont le plus mobilisées étant le Fonds de solidarité et les reports de cotisations sociales. Ce sont les entreprises dont la situation financière était médiane qui ont eu le plus recours à ces dispositifs, alors que celui-ci était plus faible pour les entreprises dont la situation était mauvaise ou excellente, I'ensemble des aides ouvertes ayant permis de réduire fortement, jusqu'à présent, le nombre des défaillances. Comparativement à ses partenaires européens, la France, dans une position médiane en ce qui concerne le volume des soutiens, a fait le choix d'une palette d'aides plus diversifiée alors que d'autres ont concentré leurs dispositifs (comme l'Allemagne qui a donné la priorité aux garanties de prêt et aux participations en capital).

Rapport de la DARES : L'activité partielle, bilan contrasté, sortie en douceur

La DARES a publié le23 avril un « focus » sur le recours à l'activité partielle de longue durée en 2020, complété d'une enquête sur les premiers mois de 2021; dispositif utilisé de façon marginale jusqu'en 2019 (de 20000 à 50000 salariés par an, principalement dans l'industrie), I'activité partielle a explosé avec la crise sanitaire en 2020 , avec un « pic » de 8,4 millions de salariés en avril, est redescendu à 1 million à l'été, avant de remonter au gré des " reconfinements » (elle se situe à un niveau encore élevé - 2,3 millions - en mai 2021). Selon l'enquête, elle s'est étendue à tous les secteurs, et d'abord aux services marchands, en particulier l'hôtellerie-restauration ( $80 \%$ des heures) qui fait l'objet d'une fermeture quasi complète depuis un an. Tous les profils sont désormais concernés, avec une forte augmentation chez les cadres, bien que ceux-ci aient plus accès au télétravail, et il apparaît que les pertes de débouchés, voire les difficultés d'approvisionnement constituent un facteur croissant de réduction d'activité. Par ailleurs, le nouveau dispositif $d^{\prime}$ " activité partielle de longue durée " (voir Repères de juin 2020) qui est destiné aux entreprises saines, 
confrontées à une forte chute d'activité (par ex Airbus), sous réserve de contreparties sur le maintien de l'emploi et la formation professionnelle, peine à démarrer, puisqu'en mars 2021, seulement 5 \% des heures chômées étaient couvertes par I'APLD; le formalisme administratif de ce régime et ses faibles avantages comparatifs par rapport à l' " activité partielle de droit commun » qui est sans cesse prorogée ont sans doute dissuadé de nombreuses entreprises éligibles. Les ministres de l'économie et du travail ont réuni les partenaires sociaux le 22 avril pour esquisser un scénario de sortie progressive : pour les salariés des secteurs non protégés, les employeurs continueraient d'avoir un reste à charge de $15 \%$ en mai, pour passer à $25 \%$ en juin et à $40 \%$ au 1 er juillet 2021 (les salariés continuant d'être indemnisés à hauteur de $84 \%$ de leur salaire net) : pour les secteurs subissant une fermeture administrative ou une très forte réduction d'activité, le reste à charge -nul actuellement- passerait à 15\% en juillet et serait aligné sur le droit commun à la rentrée.

\section{$\pi$ Lois de financement de la Sécurité sociale}

Proposition de loi organique au Sénat pour le renforcement du contrôle du Parlement sur les finances sociales

Le rapporteur général de la Commission des affaires sociales du Sénat a déposé, le 6 avril, une proposition de loi organique visant à renforcer le contrôle du Parlement sur les finances sociales. Elle comporte 4 volets principaux : faire coïncider le périmètre des LFSS avec celui des lois de programmation des finances publiques (extension à I'UNEDIC et aux régimes de retraite complémentaires; renforcer la clarté et la normativité des LFSS (extension du caractère limitatif de certaines dépenses) ; étendre le contrôle du Parlement en créant une loi d'approbation de comptes de la sécurité sociale; instaurer une « règle d'or » garantissant sur cinq ans l'équilibre des comptes de la Sécurité sociale.

\section{\ Dépenses de santé/Hôpital/ Assurance maladie}

Présentation de la deuxième étape des accords du Ségur de la Santé

Lors d'une conférence de presse le 12 avril, le ministre des solidarités et de la santé a présenté la deuxième étape des accords du Ségur de la santé, prévoyant la revalorisation des carrières pour les soignants paramédicaux (infir- miers, infirmiers spécialisés, aides-soignants, cadres de santé, masseurs kinésithérapeutes. Ces revalorisations de grille vont concerner 500000 professionnels paramédicaux et représenter un coût en année pleine de 740 ME. Elles entreront en vigueur le 1er octobre 2021 pour la fonction publique hospitalière, et début 2022 pour les fonctions publiques d'État et territoriale, ainsi que pour les personnels du secteur privé (lucratif et non lucratif), après adaptation pour ces derniers.

Le comité d'alerte de I'ONDAM prévoit un risque important de dépassement

Le comité d'alerte de l'ONDAM a produit le 15 avril une analyse des dépenses d'assurance maladie entrant dans le champ de I'ONDAM en 2020, concluant à un dépassement du quadruple de l'objectif initial, soit plus de 14 Mds€ du fait des charges liées à la crise sanitaire, dont de précédents Repères se sont déjà fait l'écho. Pour 2021, le comité d'alerte estime comme "sérieux " le risque de dépassement de l'objectif.

\section{$\lambda$ Assurance chômage}

Trou de 400 M€ dans le budget pour 2022 de Pôle emploi

Selon des informations parues dans Les Échos du 6 avril, les ressources pour 2022 de Pôle Emploi pourraient être amputées de $400 \mathrm{M} €$. Cela tient à la chute des cotisations de I'UNEDIC en 2020, qui sont prises en compte pour déterminer la dotation que le régime d'assurance chômage attribue à Pôle Emploi pour son fonctionnement deux exercices plus tard. C'est I'État qui pourrait être amené à compenser le manque à gagner.

Effets de la réforme de l'Assurance chômage

Une étude d'impact de I'UNEDIC réalisée à la demande de $\mathrm{FO}$ analyse les conséquences pour les allocataires de l'Assurance chômage de la réforme publiée le mois dernier (voir REPÈRES de mars) et qui va entrer en vigueur le 1er juillet. L'étude montre notamment les conséquences du nouveau mode de calcul du salaire journalier de référence qui sert de base à la détermination des allocations pour les nouveaux demandeurs d'emploi après le mois de juin. Bien que ces règles de calcul aient été assouplies par rapport à la précédente version du texte, la neutralisation insuffisante des périodes de chômage partiel et de congé maladie et maternité va réduire significativement l'indemnisation de ces 
nouveaux allocataires. Le décret, qui vient de faire l'objet d'un recours par les organisations syndicales devant le Conseil d'État pourrait s'en trouver fragilisé, comme l'a été la précédente version à la fin de l'année dernière, ce qui pourrait amener le gouvernement à le corriger.

\section{$\pi$ Formation et insertion professionnelle}

Nouvel effort pour l'insertion par l'activité économique en 2021

Le ministère du travail a lancé le 22 avril un nouvel appel à projet de 162,5 M€ pour l'emploi dans les structures d'insertion par l'activité économique avec un objectif de créer 30000 nouveaux emplois dans le secteur. En 2020, le financement avait été de $220 \mathrm{M} €$, permettant de maintenir les 140000 emplois existant dans le secteur et d'en financer 34000 nouveaux.

\section{$\pi$ Solidarité, lutte contre la pauvreté, revenu universel}

Rapport d'évaluation de la Stratégie nationale de prévention de lutte contre la pauvreté

Le Comité d'évaluation de la Stratégie nationale prévention et de lutte contre la pauvreté présentée par la Président de la République le 13 septembre 2018 (voir Repères de septembre 2018), qui est présidé par Louis Schweitzer, a rendu son rapport 2021 le 2 avril. Le Comité s'est d'abord penché sur l'avancement de chacune des 35 mesures de la stratégie, dans un contexte qui a changé avec la crise sanitaire; il a porté un avis contrasté sur chacun des 5 grands domaines couverts (petite enfance et éducation ; santé ; formation et emploi ; logement; droits sociaux); si plusieurs mesures ont été intégralement mises en œuvre (revalorisation de la prime d'activité, mise en œuvre de la couverture santé solidaire, renouvellement de la CSS pour les allocataires du RSA), des chantiers importants n'en sont qu'à leur début (déploiement du service public de l'insertion et de l'emploi, pour lequel 31 territoires viennent d'être sélectionnés pour une première mise en $œ u v r e$ ) ou en panne (Revenu universel d'activité) ; en revanche, les actions pour l'accès à l'emploi des jeunes et des personnes les plus éloignées de l'emploi ont été amplifiées par les mesures prises pour faire face à la crise sanitaire (extension de la Garantie jeune, mesures en faveur de l'insertion par l'activité économique). S'agissant des effets de la Stratégie sur l'évolution de la pauvreté «monétaire » proprement dite, le Comité considère qu'il est encore trop tôt pour les évaluer, et que la petite diminution $(-0,3 \%)$ de celle-ci entre 2018 et 2019 peut difficilement être imputée aux mesures de la Stratégie. Le comité soulève plusieurs points d'attention et formule 10 recommandations, dont la plus notable est l'expérimentation sans délai d'un revenu de base pour les jeunes de 18-24 ans les plus démunis. II propose par ailleurs que soit reprise la concertation sur le Revenu universel d'activité, la cohérence des deux dispositifs devant bien entendu être assurée.

\section{FISCALITÉ ET PROCÉDURE FISCALE}

\section{$\lambda$ Procédure fiscale}

Le délit caractérisé de fraude fiscale implique-t-il la démonstration de l'appréhension des sommes en causes par le fraudeur?

Selon la Cour de Cassation (Cass. crim., 8 avril 2021, Société Laboratoire Puressentiel, pourvoi $\left.n^{\circ} 19-87.905\right)$ la caractérisation du délit de fraude fiscale résultant de l'omission de déclarer les rémunérations sujettes à l'impôt sur le revenu en application de l'article 155 A du CGI n'implique pas qu'il soit démontré que le prévenu a effectivement appréhendé les sommes en causes. En l'espèce, les fondateurs d'une société ont cédé à une autre société les droits d'exploitation des marques et brevets qu'ils exploitaient. En 2013, la DGFIP a adressé aux fondateurs une proposition de rectification suite à un ESFP, considérant que l'un d'eux était le véritable gestionnaire et l'exploitant des marques et brevets cédés à la seconde société et que les redevances versées à celle-ci rémunéraient en réalité les prestations réalisées par l'un des fondateurs, qui devait être imposée à ce titre en application de l'article 155 A du CGI. En 2014, sur avis conforme de la CIF, la DGFIP a déposé plainte contre les fondateurs, qui ont été cités à comparaître devant le tribunal correctionnel pour s'être frauduleusement soustraits à l'établissement et au paiement de l'impôt sur le revenu dû au titre des années 2009, 2010 et 2011, en s'abstenant de souscrire dans les délais requis des déclarations des BNC. Les premiers juges ayant relaxé les prévenus, le procureur de la République et l'administration fiscale ont formé appel de cette décision. La Cour d'appel a précisé que les infractions reprochées ne nécessitaient pas d'établir que les fondateurs avaient directement appréhendé les fonds liti- 
gieux, leur perception pouvant être dissimulée par des structures écrans. La Cour de Cassation a rejeté le pourvoi des prévenus et confirme la conclusion de la Cour d'appel. Dès lors, la caractérisation du délit de fraude fiscale résultant de l'omission de déclarer les rémunérations sujettes à l'impôt en application de ce texte n'implique pas qu'il soit démontré que le prévenu a effectivement appréhendé les sommes en causes.

Coût budgétaire élevé des contentieux fiscaux pour l'État en 2020

Selon la Cour des comptes (voir note d'exécution budgétaire 2020 « Remboursements et dégrèvements ", avril 2021), les contentieux fiscaux ont coûté près de $6 \mathrm{Mds} €$ à l'État. L'exercice 2020 a été marqué par des décaissements particulièrement élevés de contentieux de série (1,6 Mds€ pour le contentieux OPCVM et 0,8 Mds€ pour le contentieux précompte, hors intérêts moratoires, contre respectivement 0,3 Mds€ et 0,1 Mds€ en 2019) qui expliquent la croissance de 2,3 Mds€ des remboursements des autres impôts directs. Le dénouement de ces contentieux exceptionnels, souvent anciens, explique la forte progression du versement d'intérêts moratoires particulièrement élevés (1,8 Mds€ après 0,6 Mds€ en 2019). La Cour des comptes observe un niveau proche de celui de l'exercice 2017 (6,6 Mds€) qui avait constitué un précédent avec l'imbroglio juridique lié au remboursement de la surtaxe IS de $3 \%$ aux entreprises. La sincérité des prévisions budgétaires initiales est donc contestable. La Cour des comptes recommande un enrichissement des annexes budgétaires d'informations rétrospectives relatives aux contentieux et notamment aux contentieux de série.

Crise sanitaire et moindre performance des résultats des contrôles fiscaux en 2020

La crise sanitaire a bouleversé le bon déroulement des contrôles fiscaux, notamment sur place. Le rendement budgétaire relatif à la lutte contre la fraude fiscale a été bien moins performant en 2020 avec 7,8 Mds€ de recettes contre près de 11 Mds€ en 2019, avec des contrôles fiscaux essentiellement sur pièces. Les recettes encaissées retrouvent un niveau proche de l'exercice 2018, marqué par une nette érosion des rentrées budgétaires issues de la lutte contre la fraude fiscale. Selon les éléments de réponse fournis par la DGFIP, les opérations de contrôle et les procédures de recours après contrôle ont été suspendues du mois de mars à la fin du mois de juin 2020 et n'ont repris que très progressivement à compter de juillet puis septembre 2020 .

\section{$\pi$ Impôt sur les sociétés}

Taxe GAFA : publication des commentaires définitifs au BOFIP

L'administration fiscale vient de publier au BOFIP ses commentaires relatifs à la taxe sur certains services fournis par les grandes entreprises du secteur numérique (art. 1 L. $n^{\circ} 2019$ 759 du 24 juillet 2019 ; art. 44-I-19 L. n²0201721 du 29 déc. 2020 de finances pour 2021). Ils concernent le champ d'application, le fait générateur et l'exigibilité, la base d'imposition et la liquidation de la taxe.

Associations : nouveau plafond de la franchise des impôts commerciaux

Le législateur a établi un plafond de la franchise des impôts commerciaux (IS, TVA, CET) au profit des organismes non lucratifs (art. 206-1 bis du CGI), pour les bénéfices réalisés dans le cadre de leurs activités lucratives accessoires. Trois conditions doivent être réunies : leur gestion doit être désintéressée ; les activités non lucratives doivent significativement demeurer prépondérantes et le montant de leurs recettes d'exploitation ne devait pas excéder $60000 €$. Pour 2021, ce seuil est porté à $72432 €$ pour les exercices clos à compter du 31 décembre 2020 en matière d'IS ; pour l'année 2021 en matière de CET ; pour les recettes encaissées à compter du $1^{\text {er }}$ janvier 2021 en matière de TVA.

\section{QPC transmise au Conseil constitutionnel sur l'ancienne taxe sur la publicité}

Depuis la loi de finances pour 2003, une taxe frappait la publicité diffusée à la radio et à la télévision (art. 302 bis KD du CGI). Elle a été abrogée depuis par le législateur (art. 26 L. n²0181317 du 28 déc. 2018 de finances pour 2019). La société requérante soutient que ces dispositions méconnaissaient le principe d'égalité devant les charges publiques. Le Conseil d'État vient de renvoyer au Conseil Constitutionnel la QPC concernant cette taxe dans sa rédaction initiale (CE, 20 avril 2021, SAS M6 Publicité, Req. $\left.n^{\circ} 448984\right)$. Ces dispositions auraient pour effet d'assujettir les régisseurs de messages publicitaires à une imposition dont l'assiette inclurait des sommes dont ils n'ont pas la disposition. Le Conseil d'État a considéré que cette QPC présentait un caractère sérieux, et qu'il y avait lieu de la renvoyer au Conseil constitutionnel. A suivre... 


\section{$\lambda$ Taxe sur la valeur ajoutée}

Taux de TVA à $0 \%$ sur les vaccins et tests Covid-19

Le législateur prévoit l'application d'un taux de TVA nul aux livraisons et aux prestations de services qui leur sont étroitement liées, portant sur les vaccins contre la Covid-19 bénéficiant d'une autorisation nationale ou européenne de mise sur le marché et sur les dispositifs médicaux de diagnostic de la Covid-19 (art. 46 L. n²020-1721 du 29 déc. 2020 de finances pour 2021 - art. 278 ter du (GI). L'administration fiscale vient de commenter cette mesure au BOFIP (BOI-TVALIQ-30-10-55). Le taux s'applique de manière rétroactive et temporaire aux livraisons de biens et prestations de services, y compris les importations et acquisitions intracommunautaires de biens. II s'agit des opérations dont le fait générateur intervient à compter du 15 octobre 2020 et jusqu'au 31 décembre 2022.

\section{$\lambda$ Impôt sur le revenu}

Changements intervenus en matière de déclaration d'impôt sur le revenu

La campagne de déclaration des revenus 2020 a été officiellement lancée par la DGFIP. La part de déclarations numériques est croissante et concerne trois quarts des foyers fiscaux. Les dates déclaratives limites s'étalent entre le 26 mai et le 8 juin 2020, en fonction des départements, et jusqu'au 20 mai 2020 pour la déclaration papier. La déclaration automatique est encore élargie cette année, avec une liquidation établie sur la base des informations dont la DGFIP a déjà connaissance (situation de famille, revenus, CSG déductible, dépenses d'emploi à domicile payées et PAS). Pour la déclaration de I'année 2021, il faut souligner l'exonération des frais de télétravail, de la prime exceptionnelle de pouvoir d'achat et de la prime Covid-19, utilisée dans certains établissements de santé. Les heures supplémentaires exonérées sont désormais communiquées à la DGFIP. Et les revenus issus des plateformes de l'économie collaborative sont désormais communiquées.

\section{Politique fiscale}

Stabilité de la législation fiscale du Gouvernement?

Le Gouvernement, et notamment le ministre des finances, a rappelé au mois d'avril son refus de procéder à des hausses d'impôts sur les entreprises ou sur les particuliers. En février 2021, le haut-commissaire au plan, F. Bayrou, n'avait pas exclu une contribution des contribuables les plus fortunés au financement de la dette publique née de la crise sanitaire, provoquant l'agacement des parlementaires de la commission des finances de l'Assemblée nationale qui avaient rappelé la nécessaire stabilité de la législation fiscale. Les recettes fiscales, notamment au titre de l'impôt sur le revenu et de la TVA, recouvrées en début d'année 2021, semblent plus dynamiques qu'initialement envisagées en LFI même si les incertitudes demeurent nombreuses. Le montant d'impôt sur le revenu serait supérieur de $500 \mathrm{M} €$ environ par rapport aux prévisions. Les recettes de TVA encaissées par l'État seraient aussi dynamiques qu'au début de l'année 2020 avant le confinement. Toutefois, le Gouvernement n'exclut pas le recours à une loi de finances rectificative au cours de l'été 2021, et à un décret d'avance (art. 13 LOLF), selon la déclaration du ministre des finances de ce mois d'avril 2021. Si la pratique gouvernementale observée depuis 2018 de ne plus recourir aux décrets d'avance est réellement remise en cause, ne faut-il pas alors craindre le retour des dispositions fiscales en loi de finances rectificative, et la fin de la stabilité de la législation fiscale?

\section{Management PUblic}

\section{$\pi$ Administration numérique}

Périmètre étendu pour l'échange de données entre administrations

Le décret n 2021-464 du 16 avril 2021 étendant le champ des échanges d'informations et de données entre administrations dans le cadre des démarches administratives a été publié au Journal officiel du 18 avril 2021. Pris en application de la loi nº 2018-727 du 10 août 2018 pour un État au service d'une société de confiance, le texte élargit la liste des organismes et des informations concernés par l'échange de données entre administrations. Sont donc désormais concernés : le recensement citoyen obligatoire ; la situation de la famille, des personnes scolarisées et des demandeurs d'emploi ; les revenus des particuliers; les diplômes, titres et qualifications professionnelles détenus.

\section{Collectivités territoriales}

\section{Simplification des expérimentations lo-} cales

Le Conseil constitutionnel a validé le 15 avril 2021 (décision 2021-816 DC) la loi organique relative à la simplification des expérimentations mises en œuvre sur le fondement du qua- 
trième alinéa de l'article 72 de la Constitution. Celle-ci avait été adoptée le 16 mars 2021 par le Parlement suite à un vote conforme par les députés du texte adopté en première lecture par le Sénat le 3 novembre 2020. La loi (dossier complet) prévoit notamment : de simplifier la procédure d'entrée dans l'expérimentation ; $d^{\prime}$ 'assurer une évaluation plus pertinente des expérimentations; de permettre la sortie de l'alternative binaire entre la généralisation ou l'abandon de l'expérimentation.

Néanmoins, rappelant le principe d'égalité, le Conseil constitutionnel précise que le législateur " ne saurait maintenir à titre pérenne des mesures prises à titre expérimental dans les seules collectivités territoriales ayant participé à l'expérimentation sans les étendre aux autres collectivités présentant les mêmes caractéristiques justifiant qu'il soit dérogé au droit commun».

\section{$\pi$ Fonction publique}

\section{Égalité des chances et haute fonction pu- blique}

Afin de favoriser l'égalité des chances et la diversification du recrutement dans la fonction publique, une ordonnance et un décret du 3 mars 2021 créent, à titre expérimental, des concours spéciaux d'accès aux jeunes de milieux défavorisés (dossier complet). Les établissements concernés sont l'École nationale d'administration, I'Institut national d'études territoriales, l'École des hautes études en santé publique, l'École nationale supérieure de la police et l'École nationale d'administration pénitentiaire.

Une commission d'admission est en charge de la sélection des candidats au regard notamment de la qualité de leur parcours de formation antérieur, de leurs aptitudes et de leur motivation. La sélection comprend l'examen des dossiers des candidats et un entretien.

Cette expérimentation devra faire l'objet d'une évaluation avant fin juin 2024. Parallèlement, une refonte de l'ensemble des concours d'accès aux fonctions publiques devra être lancée, destinée à garantir le caractère non discriminatoire des modalités de sélection tout en garantissant la bonne adéquation des recrutements aux différents besoins des administrations.

\section{Réforme des inspections générales}

Le Gouvernement a transmis aux organisations syndicales le 27 avril 2021 l'article 7 du projet d'ordonnance de réforme de la haute fonction publique. Ce texte, relatif aux inspections générales, prévoit la mise en extinction des trois corps d'inspection : inspection générale des finances (IGF), inspection générale de l'administration (IGA) et inspection générale des affaires sociales (IGAS). De manière indirecte, il s'agit ainsi d'instaurer une "fonctionnalisation » des différents corps d'inspection dans lesquels les nominations ne s'opéreraient plus à vie, mais pour une durée limitée. L'objectif de cette réforme est de favoriser les mobilités entre fonctions publique ou secteur privé, tout en renouvelant les compétences des membres de ces services.

\section{EUROPE}

\section{$\pi$ Politique européenne}

\section{Aides d'État à finalité régionale : nouvelles lignes directrices}

Le 19 avril, la Commission européenne a adopté de nouvelles lignes directrices relatives aux aides d'État à finalité régionale, qui permettent de réduire les écarts de développements entre régions. Ces nouvelles dispositions ont pour but principal de réduire la complexité du régime et surtout de mieux intégrer les critères environnementaux au processus d'admission de telles aides. Concernant la politique conjoncturelle de large acceptation des aides d'État ayant pour finalité la lutte contre les conséquences de la crise sanitaire actuelle, elle se poursuit tout au long du mois d'avril avec notamment, le 6 avril, I'admission d'une restructuration française de près de 4 Mds€ en faveur d'Air France.

Mécanisme pour une transition juste : facilité de prêt au secteur public

Le 26 avril, le Parlement européen et le Conseil se sont mis d'accord sur la proposition de la Commission relative à une facilité de prêt au secteur public. Comme pour toutes les propositions du mécanisme pour une transition juste, il ne manque plus que l'approbation finale du Parlement européen et du Conseil. Cette facilité, constituée d'une combinaison de subventions (1,5 Mds€) financées par le budget de I'UE et de prêts (10 Mds€) accordés par la Banque européenne d'investissement (BEI), en faveur des projets qui ne génèrent pas suffisamment de recettes pour être financièrement viables, relève du mécanisme pour une transition juste (MTJ), destiné à mobiliser au moins $150 \mathrm{Mds} €$ sur la période 2021-2027 dans les régions les plus touchées, afin d'atténuer l'impact socio-économique de la transition climatique. 


\section{त Budget européen}

NextGenerationEU : adoption d'une stratégie de financement diversifiée

Le 14 avril, la Commission européenne a adopté une stratégie de financement diversifiée pour lever, jusqu'en 2026, sur les marchés au meilleur prix les 800 Mds€ d'emprunts prévus par le plan de relance intitulé NextGenerationEU. II faut maintenant à la Commission mettre en place un réseau des spécialistes en titres publics puis publier la première décision d'emprunt annuelle et le premier plan de financement (qui sera mis à jour deux fois par an). Quant aux États membres, ils sont invités à présenter leurs plans pour la reprise et la résilience avant le 30 avril, mais ils peuvent le faire à tout moment jusqu'à la mi-2022. En pratique, si quelques-uns ont commencé juste avant (le Portugal en premier le 22 avril, l'Allemagne et la Grèce le 28 avril, la France et la Slovaquie le 29), les autres suivent immédiatement début mai.

Analyse sur les contributions financières de pays tiers à l'UE

Le 27 avril 2021, la Cour des comptes européenne a publié la première analyse d'ensemble des contributions financières de 18 pays tiers versées à l'UE et à ses États membres, ce qui représente environ $1 \%$ des recettes de I'UE et quelque $7 \mathrm{Mds} €$ au cours de la période 2014 2019. Les plus grands contributeurs ont été la Suisse et la Norvège (2,2 Mds€ chacune), la Turquie (1,3 Md€) et Israël (1,0 Md€). En contrepartie, les pays tiers ont accès à certains programmes ou activités de l'UE tels qu'Horizon 2020 ou Erasmus+. Outre ces contributions versées à I'Union, les quatre États de l'AELE (Islande, Liechtenstein, Norvège et Suisse) versent chaque année en moyenne un demi-Md€ directement à certains États membres de I'UE au titre de la politique de cohésion ainsi élargie à I’Espace économique européen.

Fonds structurels : les dépenses ont doublé en 2019-2020

Selon un rapport publié le 28 avril 2021 par la Commission, le taux d'investissement des cinq Fonds structurels et d'investissement européens (Fonds ESI) dans I'UE a considérablement augmenté, passant de $28 \%$ de l'enveloppe totale en 2018 à $56 \%$ à la fin de 2020 . Cette évolution est en partie le résultat de la flexibilité, introduite dans la législation relative à la politique de cohésion, qui est procurée par les initiatives $d$ 'investissement en réaction au coronavirus.

\section{INTERNATIONAL}

\section{$\lambda$ Fiscalité}

Les États-Unis en faveur d'un taux minimal d'IS

À la veille d'une rencontre virtuelle des grands argentiers du G20, mercredi 7 avril, la Secrétaire américaine au Trésor, et ancienne présidente de la Federal Reserve, Janet Yellen, s'est engagée, au nom de l'administration Biden, en faveur de l'instauration d'un taux minimum mondial pour l'imposition des sociétés. II s'agit d'un revirement complet des États-Unis qui rend désormais crédible l'adoption de cette mesure prônée par l'OCDE. Son mécanisme consisterait pour les pays concernés à imposer les multinationales installées dans des paradis fiscaux de la différence entre leur taux d'IS et ce taux minimal mondial. L'impôt ne dépendrait ainsi plus du lieu d'établissement mais des bénéfices réalisés dans chaque pays. Cette prise de position, saluée notamment en Europe par la France et l'Allemagne, permettrait de véritablement lancer la nouvelle direction de l'OCDE confiée le 12 mars à Mathias Cormann, ancien ministre des finances et député australien qui ne s'était jusqu'alors pas signalé par son opposition à la fraude fiscale. Pascal Saint-Amans, directeur des questions fiscales à l'OCDE doute que le seuil finalement adopté soit de $21 \%$ mais a bon espoir qu'il s'en rapproche et soit loin des $12,5 \%$ qui étaient initialement discutés (et correspondent au taux de I'IS en Irlande).

Aurélien BAUDU (Fiscalité et procédure fiscale), Fabrice BIN (Europe, international), Jean-François CALMETTE (budget de l'État et des opérateurs),

Matthieu HOUSER (finances locales), Michel LE CLAINCHE (comptabilité publique, management public, coordination), Yves TERRASSE (finances sociales), Robin DEGRON (coordination).

Retrouvez sur www.rgfp.fr:

- I'intégralité des REPÈRES d'actualité des finances et de la gestion publiques depuis 2015

- le 15 du mois, les REPÈRES d'actualité du mois précédent. 